\title{
Novel Mechanical Design of an Ear Surgical Device to Treat Otitis Media with Effusion
}

\author{
Cailin Ng${ }^{1}$, Wenyu Liang ${ }^{2}$, Chee Wee Gan ${ }^{3}$, Hsueh Yee Lim ${ }^{3}$, Kok Kiong Tan ${ }^{2}$ \\ ${ }^{1}$ NUS Graduate School of Engineering and Integrative Sciences, National University of Singapore \\ Singapore \\ cailin.ng@u.nus.edu \\ ${ }^{2}$ Department of Electrical and Computer Engineering, National University of Singapore \\ Singapore \\ ${ }^{3}$ Department of Otolaryngology, National University of Singapore \\ Singapore
}

\begin{abstract}
Otitis Media with Effusion is an ear disease that requires a surgery to be performed under general anesthesia (GA) to place a grommet in the tympanic membrane for severe cases. An automated grommet insertion device, the Ventilator Tube Applicator (VTA) enables the grommet insertion surgery to be completed in a short time automatically and precisely by automating the incision and insertion process. However, its current design limits the usefulness of the device as it is restricted by the properties of the eardrum, such as the angle, thickness and strength. Therefore, a cover-cutter toolset design with three different setups was conceptualized. Experimental results on mock membrane showed that the new toolset design increased the surgical device's success rate with a lower force on the tympanic membrane (TM), hence improving the surgical device's overall efficiency and safety.
\end{abstract}

Keywords: Otitis Media with Effusion, Medical Device, Mechanical Design.

\section{Introduction}

Otitis media with effusion (OME) is a very common ear disease that causes body imbalance, discomfort and may even result in irreversible damage to the middle ear structure. When the Eustachian tube is dysfunctional, fluid will accumulate in the middle ear space and cause discomfort and loss of hearing. OME affects most commonly infants and children, although it may also affect people of all ages worldwide. When antibiotics as a treatment for OME fails, a grommet is surgically inserted on the tympanic membrane (TM) so that the accumulated fluid can be drained out [1]. The surgery starts with myringotomy, where the surgeon makes a small incision on the TM with a myringotomy knife. The surgeon then performs suction to remove the middle ear fluid behind the TM, before pushing a grommet into the incision either by using the forceps or a pick. Overall, the surgery involves a number of steps that takes around 15 - 30 minutes to complete and utilizes 3 to 4 main tools - myringotomy knife, suction tube, forceps and/or pick. This surgery can be performed under local anesthesia (LA) in adults if they can tolerate the discomfort. However, the usual practice for young children is to use general anesthesia (GA) to keep them absolutely still during the procedure to prevent accidental damage to the internal ear structure. Currently, the insertion of grommet is one of the most common paediatric surgery performed and cited as the most common reason for a child to undergo a GA $[2,3]$. Studies have shown that there are long term health effects from GA, including possible delay in brain development of children $[4,5]$.

A novel "all-in-one" device, known as the Ventilation Tube Applicator (VTA), allows office-based myringotomy with grommet insertion for an OME patient [6]. Experimental results on mock membrane showed that the prototype is able to achieve a success rate of $98 \%$. The VTA utilizes a highly integrated structure encompassing key components of a motorized mechanical system, a sensing system and a motion control system. With the sensing system in VTA that notifies the surgeon when the toolset touches the TM, and the ultrasonic piezomotor that controls the actuation, the VTA is able to complete the surgery in a short time automatically and precisely. The prototype has since been improved slightly to reduce its bulk, weight, operation time and cost, but the general working process and toolset remains unchanged. Fig. 1 shows the working process of the VTA, Fig. 2 shows the VTA with its control system, and Fig. 3 shows the distal end of the toolset with a pediatric grommet, Tiny Tytan (Medtronic pdt ID 1056101) loaded axially on the toolset. The cylindrical needle-like cutter (needle- 
cutter) supports the grommet by holding it in place, and during incision, cuts the TM by punching out through the bore of the grommet. The needle-cutter's diameter is slightly smaller than the inner diameter of the grommet such that it is able to slot through the bore of the grommet. Once an incision is made by the needle-cutter, the holder then pushes the grommet until it is inserted into the incision. Although this is effective, as shown in the high success rate of insertion on mock membrane, it is somewhat limited. This is because a change in the mock membrane material, thickness and the angle of toolset approach might affect its success rate. The VTA will need to overcome these challenges before entering the clinical trial phase.

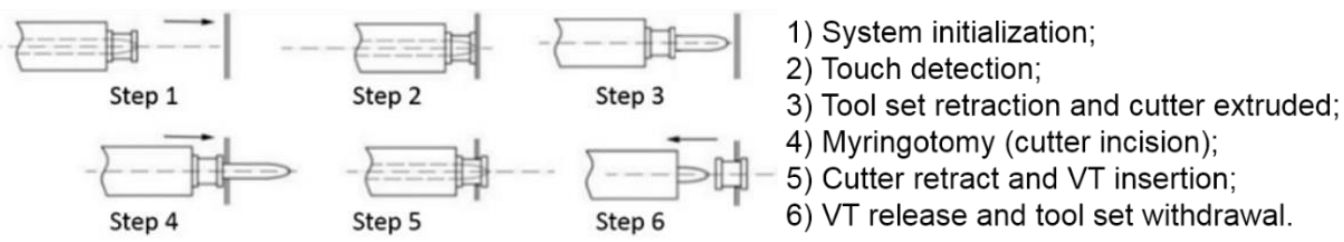

Fig. 1: Working process of the VTA [6].

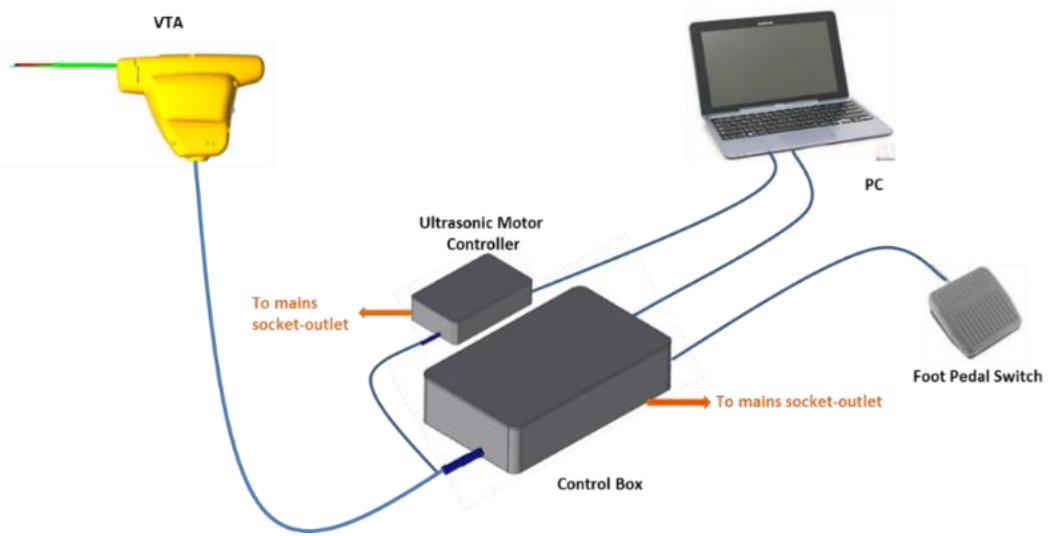

Fig. 2: VTA and its control system [6].

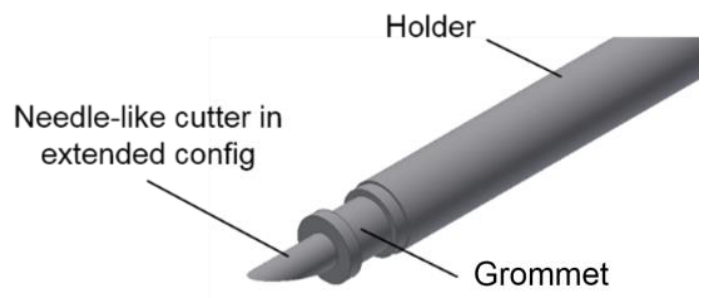

Fig. 3: Distal end of the toolset, the needle-cutter [6].

The contribution of this paper is to introduce a novel method to improve the current design of the VTA so as to overcome the limitations of the surgical device. This paper is organized as follows: Section II describes the challenges of VTA. Section III introduces and discusses the new mechatronic design. Section IV presents the experimental results on mock membrane. Section V concludes this report and Section VI describes the future work of this research.

\section{Challenges of the VTA}

\subsection{TM Angle}

A challenge of the current VTA design is that the human TM is usually at an oblique angle. Although the success rate of VTA on mock membrane is $98 \%$, it may not be indicative of the clinical success rate on humans. This is because the experiment setup in the laboratory utilized an imperfect TM model [9] as a first step to verify capability of the device. The TM model is formed by slightly stretching a mock membrane over the opening of an $8 \mathrm{~mm}$ diameter cylinder. The cylinder 
is shaped regularly, hence the mock membrane forms a vertical plane. During the experiments, the VTA approaches the mock membrane horizontally, forming a near $90^{\circ}$ approach with the mock membrane. In reality, the angular placement of the TM within the ear canal may range between $45^{\circ}$ to $60^{\circ}$ for adults [7], and may even be in an extremely oblique angle for neonate and infants [8]. An experiment was done to measure the success rate of VTA with needle-cutter on mock membrane at different angles. The results, as shown in Fig. 4, are not satisfactory. The success rate of VTA with needle-cutter falls to $40 \%$ if the mock membrane is placed at a $50^{\circ}$ angle and it is unable to insert the grommet if the mock membrane's angle is lower than $50^{\circ}$. This is because of a few reasons:

- Geometrical shape of the grommet not suited for $0^{\circ} \mathrm{z}$-direction insertion for very oblique TM,

- Grommet sliding away from the incised hole because of the angle of the oblique surface, and

- High speed insertion causing the grommet to tilt excessively on the oblique surface, hence it is not in the correct position for insertion.

TM angle is an important factor as statistics have shown that OME is more prevalent in toddlers aged 3 and below [4], and for this age group, their TM is likely to be oblique. To make VTA useful as a treatment tool for OME, it has to overcome the oblique TM issue.

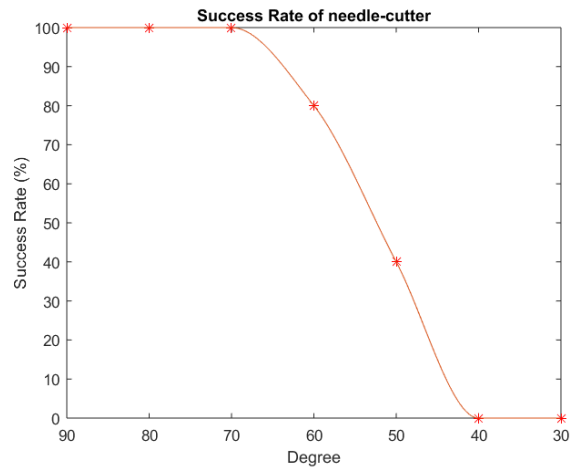

Fig. 4: Success rate of VTA on different membrane angles.

\subsection{TM Variability}

The human TM is a thin cone-shaped membrane that separates the external ear from the middle ear. It is made up of three layers of tissue: the outer cutaneous layer, the fibrous middle layer and a mucous inner membrane, and held in place by a thick cartilage ring. The TM is approximately $0.08 \mathrm{~mm}$ thick, and $10 \mathrm{~mm}$ in diameter. However, the TM thickness, strength and flexibility varies between individuals. In [10], the thickness distribution of six human TMs is taken using a highresolution optical coherence tomography setup. It was found that the thickness varies strongly across each membrane and a great deal of inter-specimen variability can be seen. Hence, the VTA needs to be able to adapt to different TM thickness. The current VTA design inserts the Tiny Tytan grommet by incising the TM using a $0.76 \mathrm{~mm}$ diameter needle-cutter, and pushing a $1.5 \mathrm{~mm}$ diameter Tiny Tytan grommet into the incision. Since the diameter of the incision is much smaller than that of the width of the grommet, it is possible that the VTA might need a considerable force to push the grommet through the incision if the TM is thicker or stiffer. The success rate might be affected by the properties of the TM, and that is not acceptable for a surgical device.

\section{Method}

Our objective was to improve the VTA such that it can be used on a bigger range of TM angles and to increase its success rate on different type and thickness of mock membrane. There are several ways to so this, for example to rework VTA so that an additional actuation axis can be added to allow more degree of freedom to manoeuvre the toolset. Another way is to optimise the control parameters for the current piezomotor setting. The former would result in a very bulky and costly device that is not suitable for a handheld surgical device, and the latter did improve the performance but was still not sufficient [9]. We came up with a novel design for the toolset that would not require any change in the actuation methods, and yet achieve our objective. 


\subsection{Toolset Design}

We identified that the fundamental problem is that the incision made by the needle-cutter is too small and the grommet slides away from the incision if the TM is oblique. Hence, the idea of a new toolset design, the cover-cutter, was conceptualized. The needle-cutter is designed such that the needle-like cutter is contained within the hollow of the holder and passes through the bore of the grommet when protruded, hence the diameter of the cutter is limited to the diameter of the inner bore of the grommet, which is $0.76 \mathrm{~mm}$. To overcome this limitation, the cover-cutter is designed such that it is on the exterior of the holder and positioned so that its cutting blade is on the same side of the sliding motion. The cover-cutter is a cylinder that sheathes the holder, as shown in Fig 5a, and is used to make an incision in the TM. The front portion of the cover-cutter is a partial cylinder with a sharp blade tip, measuring $1.6 \mathrm{~mm}$ in arc length. The holder has a conical tip to support the grommet in an axial position similar to the needle-cutter. Fig $5 \mathrm{~b}$ shows the toolset setup with the grommet loaded onto the holder. The holder also acts as a pusher to insert the grommet into the incision, with its conical tip allowing the grommet to tilt slightly in any direction. As shown in Fig 5c, the incision made by the cover-cutter is a crescent-shaped slit that follows the contour of the round inner flange of the Tiny Tytan grommet.
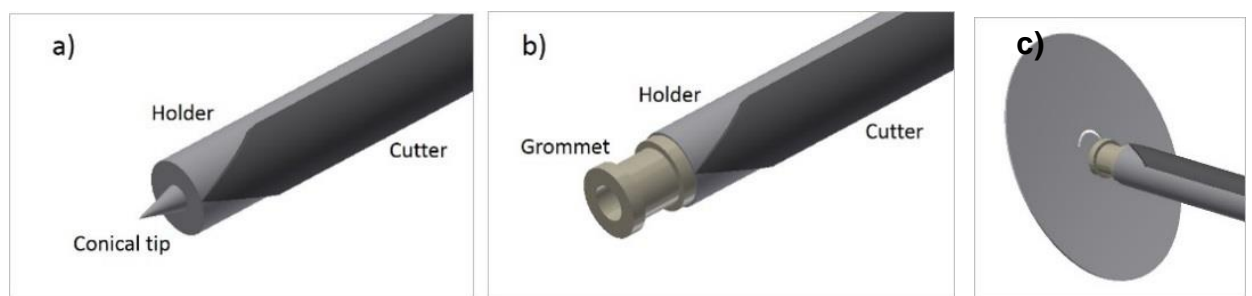

Fig. 5: CAD model of the (a) complete setup of cover-cutter with conical tip and cutter at the outer sheath of the holder; (b) with grommet loaded axially; (c) crescent shaped incision made in the mock membrane by the cover-cutter.

As opposed to the z-direction insertion by the needle-cutter method, the cover-cutter method makes use of the oblique angle of the TM as well as the inner holder with a conical tip to assist in the sliding motion of the grommet for insertion. When an object is pushed against an oblique surface, it naturally slides in the direction of the slope. Since the human eardrum is typically inclined at an angle of around $30^{\circ}-60^{\circ}$, the cover-cutter method makes use of this slope to create a sliding action to weave the loosely-held grommet into the incision. We apply a topical anesthetic gel (Lidocaine $2 \%$ ) on the grommet to ease the pain as well as to aid the sliding and weaving-in motion of the grommet from the lubricating effect of the anesthetic gel. Coupled with a crescent-shaped slit that follows the contour of the flange of the grommet, it will be able to weave into the slit easily with minimal effort. The cover-cutter method created a natural three degree of freedom (DOF) motion, even though the motor only had 1 DOF in the z-direction. With a wider cutting blade and a 3-directional motion of the grommet, the success rate of the procedure is increased for oblique TM angles.

The working process of the VTA with cover-cutter design is very similar to that of the needle-cutter and involves the following five steps as illustrated in Fig 6.

- Initialization: surgeon starts up the VTA program to initialize VTA and controller. Surgeon then inserts the distal end of the toolset with grommet into the ear canal of patient and slowly approaches the TM until it is close but not yet touching the TM.

- Touch detection: surgeon steps on the foot pedal to start the VTA operation. The toolset, actuated by the device's motor, will move forward slowly until the force sensor detects a touch.

- Myringotomy: an actuator punches out the cover-cutter to make an incision on the TM. After an incision is made, the cover-cutter is retracted.

- Grommet insertion: the motor will move the toolset forward for insertion. Because the TM is oblique, and the inner tip is shaped conically, the grommet tilts slightly in the direction of the TM's slope and slides into the incision previously made by the cover-cutter.

- Grommet release: upon the force sensor sensing a successful insertion, the motor withdraws the toolset. A buzzer will sound, signaling the end of the procedure. The surgeon can then pull the toolset out of the ear canal. 


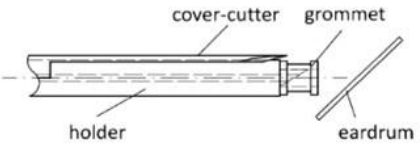

(a) initialization

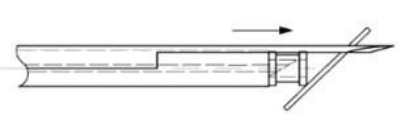

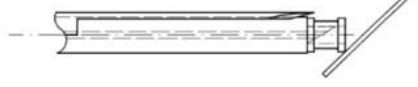

(b) touch detection

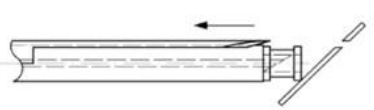

(c) myringotomy

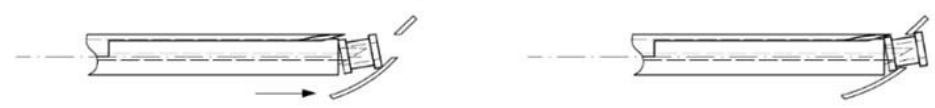

(d) grommet insertion

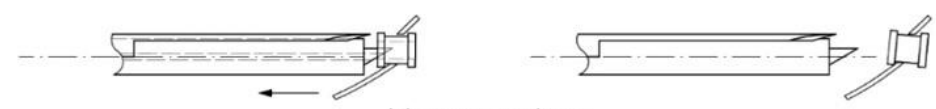

(e) grommet release

Fig. 6: Working process of the VTA with cover-cutter.

\subsection{Different Cover-cutter Setups According to TM angle}

With the cover-cutter toolset, the insertion is facilitated by the weaving-in slide of the grommet into the incision with an oblique TM. However, the anatomy of TM differs between individuals. Hence, it is possible to have a patient whose TM falls outside of the normal TM range of angles $\left(30^{\circ}-60^{\circ}\right)$. For a TM which is less oblique and forms a near $90^{\circ}$ with respect to the central axis of the ear canal, there will be very little sliding action; and for TM which is very oblique such that the angle is less than $45^{\circ}$, the grommet will tilt too much until the sliding becomes unstable and the grommet misses the incision hole. A possible solution is to have a different toolset for three different cases: less oblique TM, normal TM and very oblique TM. The distal end of the holder where the grommet is placed is slanted at an angle of $30^{\circ}$ such that the grommet will be tilted at an angle during the insertion procedure. This simple modification to the holder allows angular compensation so that the grommet will always be in an optimal position. Once the surgeon has identified his patient's TM angle: less oblique, normal or very oblique, he can then use the corresponding setups; S1, normal or S2 respectively, as shown in Fig. 8.

a)

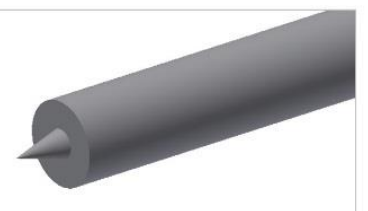

b)

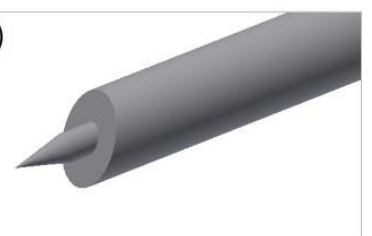

Fig. 7: Distal end of the (a) normal cover-cutter holder for normal TM angles, (b) slanted cover-cutter otherwise.

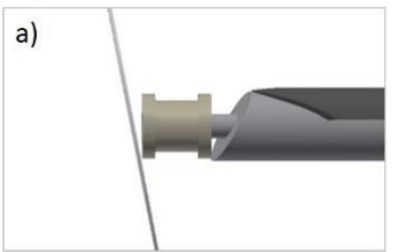

Fig. 8: Three setups for the (a)

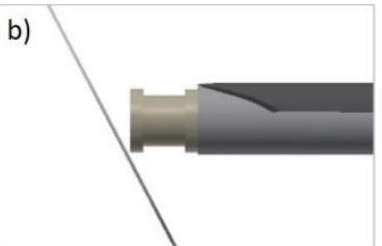

(a) less oblique,

(b) normal, (c) very oblique TM angles.

\section{Results}

The comparison between the success rate and force on the membrane between needle-cutter and cover-cutter is performed. Different membrane materials were also sourced for this experiment to test the toolset's robustness to TM variability. As explained earlier, the TM thickness and its strength and flexibility varies between individuals. Therefore, it is necessary to test the performance of the needle-cutter and cover-cutter on mock membrane of different thickness and materials of different tensile strength. Four different mock membranes were used, as shown in Table I. Mock membrane 1 is 
made of soft PVC material and is very thin. Mock membrane 2 is made of PE material with normal thickness. Mock membrane 3 is also made of PE material but is slightly thicker than Mock membrane 2. Mock membrane 4 is significantly thicker than the others as it is made up of 2 layers, with the first layer made of PE and the second layer made of latex adhesive. This wrap is made to be self-adhesive that is activated by compression, hence the second layer consist of a series of protrusions in a regular pattern. Mock membrane 4 was chosen although it is much thicker than the rest as the human TM has an array of collagen fibers in the radial and circumferential direction, and the protrusions on mock membrane 4 bear a slight resemblance to it. The experiments were repeated for membrane angles $90^{\circ}$ to $30^{\circ}$, and for the four different membrane materials.

Table 1: Different types of mock membrane used.

\begin{tabular}{|c|c|c|}
\hline Membrane & Thickness & Material \\
\hline 1 & Thin & PVC \\
\hline 2 & Regular & PE \\
\hline 3 & Thick & PE \\
\hline 4 & 2 Layers & PE and latex adhesive \\
\hline
\end{tabular}

The results as shown in Fig. 9 and 10 exhibit a vast improvement in the success rate for oblique membrane angles and across all different types of membrane. Angles that was previously unsuccessful with the needle-cutter, is now possible using the cover-cutter. The success rate of the needle-cutter is also greatly affected by the thickness and material of the mock membrane, whereas it is less so for the cover-cutter. This might be because the needle-cutter requires a larger force to push the grommet into a small incision. The insertion depth required may also have exceeded the maximum insertion depth for safety purpose. The insertion force of cover-cutter is lower across the board, with up to $78 \%$ improvement in force reading as compared to the needle-cutter. However, due to the wider cutting edge of the cutter, the incision force of the cover-cutter is higher than that of the needle-cutter. Therefore, for a fair comparison, the overall peak force, $f_{\text {peak }}$ is considered. The average improvement in peak force is around $14 \%$ for the cover-cutter.
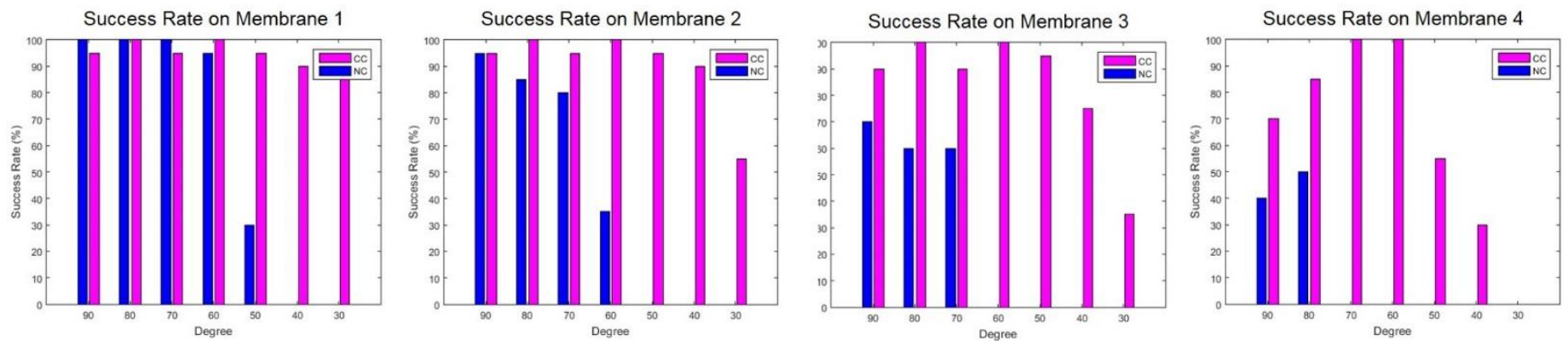

Fig. 9: Success rate comparison between needle-cutter and cover-cutter for the four types of membranes.
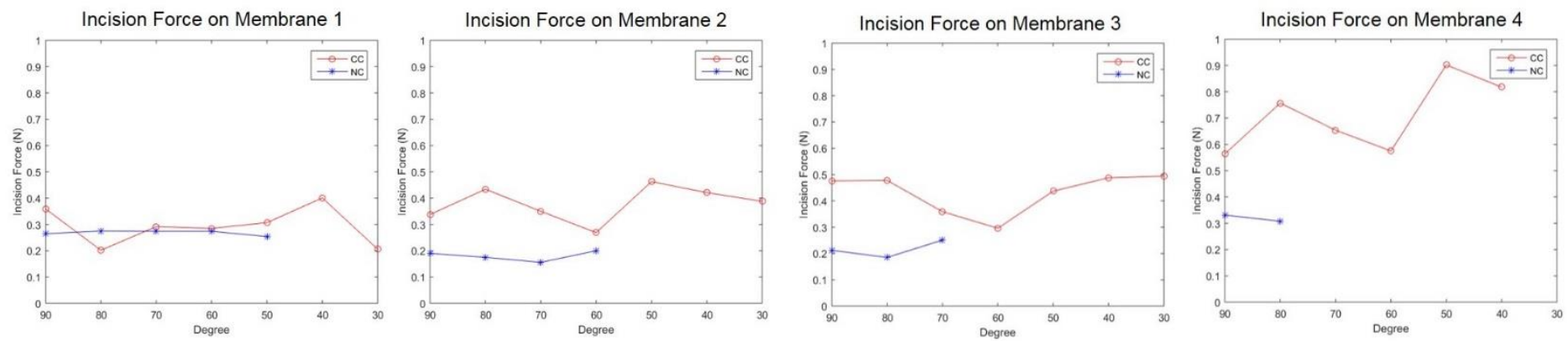

Fig. 10: Incision force comparison for the four types of membranes. 

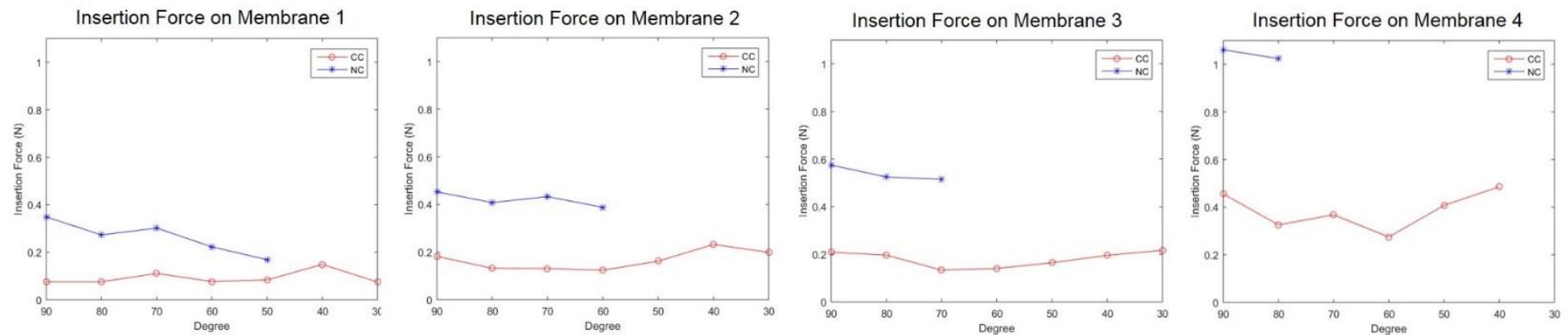

Fig. 11: Insertion force comparison for the four types of membranes.
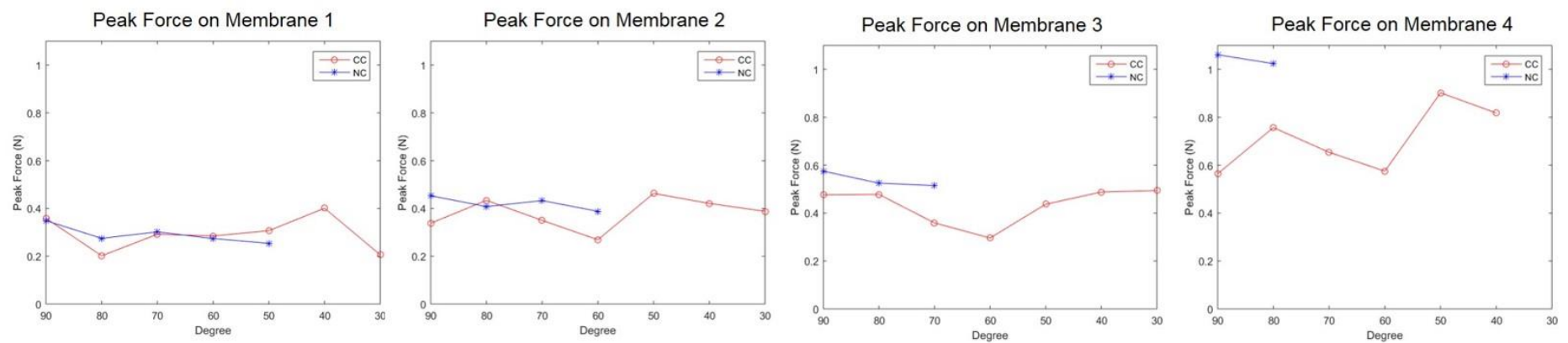

Fig. 12: Peak force (maximum of incision force and insertion force) comparison for the four types of membrane.

\section{Conclusion}

The VTA with its motorized mechanical system and a sensing system offers an automated electromechanical solution that is able to complete the myringotomy and grommet insertion procedure in a short time efficiently and precisely. To overcome its existing challenges, the cover-cutter design with three different setups was conceptualized. Experimental results on mock membrane showed that the cover-cutter design has great improvement over needle-cutter design in terms of success rate and insertion force. Very oblique angles that the needle-cutter has previously failed is now possible with the covercutter. The cover-cutter also proved to be less susceptible to variability in the types of mock membrane as it maintained a high success rate for the four different types of mock membrane used, unlike the needle-cutter. The novel design of the toolset has played a significant role in improving the performance of the device. By making use of the natural sliding motion of the grommet on the oblique surface, the cover-cutter design has made it possible for VTA to overcome its existing challenges without any major rework on its electromechanical setup, and moved one step closer from a lab prototype towards clinical trials.

\section{Future Work}

The cover-cutter design offers huge advantages over the needle-cutter, but it is not without its shortcomings. Firstly, the neonatal TM angle is near horizontal, but the cover-cutter's success rate drops drastically for angle less than $40^{\circ}$. More work needs to be done to facilitate an even larger range of TM angles. Secondly, the cover-cutter design requires some knowledge of the TM angle. Hence, a device that is able to capture and create a 3D image of the desired grommet insertion site will be helpful to minimize judgement errors that might result in lower success rate. The current 3D modeling techniques are quite advanced, but to be able to capture a 3D image of an $8 \mathrm{~mm}$ by $8 \mathrm{~mm}$ TM might be challenging. To add to the difficulty, the device has to go through a narrow and tortuous ear canal before it can be near enough to the TM to get a good image, hence it has to be very small.

\section{Acknowledgements}

This research is supported by the Science and Engineering Research Council within the Agency for Science, Technology and Research through the Biomedical Engineering Programme under Grant 1321480014. 


\section{References}

[1] J. L. Paradise, H. E. Rockette, D. K. Colborn et al., "Otitis media in 2253 Pittsburgh area infants: prevalence and risk factors during the first two years of life," in Pediatrics, vol. 99, pp. 318-333, 1997.

[2] G. C. Ilechukwu, et al., "Otitis Media in Children: Review Article," in Open Journal of Pediatrics, vol. 4, pp. 47-53, 2014.

[3] L. Vaile, T. Williamson, A. Waddell, G. Taylor, "Interventions for ear discharge associated with grommets (ventilation tubes)," in Cochrane Database Syst Rev, vol. 2, CD001933, 2006.

[4] P. Auernger, et al., "Trends in OME in America," Pediatrics, vol. 112, pp. 514-520, 2003.

[5] C. DiMaggio, et al, "A retrospective cohort study of the association of anesthesia and hernia repair surgery with behavioral and developmental disorders in young children," in J Neurosurg Anesthesiol, vol. 21, pp. 286-91, 2009.

[6] K. K. Tan, W. Liang, S. Huang, L. P. Pham, H. Y. Lim and C. W. Gan. "Design of a surgical device for office-based myringotomy and grommet insertion for patients with otitis media with effusion," in Journal of Medical Devices, vol. 8, p. 031001, 2014.

[7] J. P. Fay, S. Puria, C. R. Steel, "The discordant eardrum," in Proceedings of the National Academy of Sciences, vol. 103, pp. 19743-19748, 2006.

[8] C. D. Bluestone, J. O. Klein, "Otitis Media in Infants and Children," 4th ed, BC Decker, 2007.

[9] C. Ng, W. Liang, W. Wu, C. W. Gan, K. K. Tan, "Pain mitigation approach in office-based procedure: case study," in 42nd Annual Conf of the IEEE, pp. 601-606, 2016.

[10] Van der Jeught et al, "Full-field thickness distribution of human tympanic membrane obtained with optical coherence tomography," in JARO, vol. 14, no. 4, pp. 483-494, 2013. 